\title{
Active Structural Acoustic Control of Clamped Flat Plates Using a Weighted Sum of Spatial Gradients
}

\author{
William R. Johnson, ${ }^{1}$ Daniel R. Hendricks, ${ }^{1}$ \\ Scott D. Sommerfeldt, ${ }^{2}$ and Jonathan D. Blotter ${ }^{1}$ \\ ${ }^{1}$ Department of Mechanical Engineering, Brigham Young University, Provo, UT 84602, USA \\ ${ }^{2}$ Department of Physics and Astronomy, Brigham Young University, Provo, UT 84602, USA \\ Correspondence should be addressed to Jonathan D. Blotter; jblotter@byu.edu
}

Received 13 October 2014; Revised 6 January 2015; Accepted 7 January 2015

Academic Editor: Mohammad Elahinia

Copyright (C) 2015 William R. Johnson et al. This is an open access article distributed under the Creative Commons Attribution License, which permits unrestricted use, distribution, and reproduction in any medium, provided the original work is properly cited.

The weighted sum of spatial gradients (WSSG) control minimization parameter is developed for use in active structural acoustic control (ASAC) on a clamped flat rectangular plate. The WSSG minimization parameter is measured using four accelerometers grouped closely together on the test structure. In previous work, WSSG was developed on a simply supported flat rectangular plate and showed promise as a control metric. The displacement on the clamped plate has been modeled using an approximate analytical solution assuming shape functions corresponding to clamped-clamped beams. From the analytical formulation, weights, which were found to be the reciprocal of the wave number squared, have been derived to produce a uniform WSSG field across the plate. In active control simulations, this quantity has been shown to provide better global control of acoustic radiation than volume velocity. Analysis is presented which shows that comparable control, regardless of the sensor location, can be achieved using WSSG. Experimental results are presented which demonstrate that WSSG works effectively in practice, with results similar to the simulations. The results show that minimization of WSSG can be used as an effective control objective on clamped rectangular plates to achieve attenuation of acoustic radiation.

\section{Introduction}

Active structural acoustic control (ASAC) is an important subfield of active noise control (ANC). In ASAC, sound fields created by radiating structures are controlled using a control actuator applied to the vibrating structure as opposed to being placed in the sound field. In the late 1980s, research showed that only one or two control forces applied properly to the structure, as opposed to multiple actuators placed in the sound field, could often provide global sound reduction $[1,2]$. By moving the actuator to the surface of the plate, significant global reduction, by as much as $40 \mathrm{~dB}$ in some cases, was achieved. It was shown that only one or two actuators were needed to provide control regardless of the frequency of excitation. This was due to the fact that only one to two structural modes seemed to contribute to the radiated sound at a given frequency [3].
Another important contribution to ASAC was the development of the radiation resistance matrix method which provided an effective way to calculate the radiated sound power of a distributed system [4]. One of the key analytical tools in the development of ASAC was derived from the radiation resistance matrix, namely, the acoustic or radiation modes of a structure, which correspond to the eigenvectors of the radiation resistance matrix. The radiation modes are a set of orthogonal modes that form a set of basic functions for the acoustic field and radiate sound power independently. Each radiation mode is composed of several contributing structural modes and can be used to show which structural modes are significant in the radiation of sound power [5].

For rectangular plates it was shown that for values of $k l<$ 0.5 , where $k$ is the wave number and $l$ is the length of the longer side of the plate, the first radiation mode is the only radiation mode which significantly radiates. The shape of this 
first mode is comparable to volume velocity and it suggested that using an error sensor to measure volume velocity on the plate would be an effective way to control radiated sound [5]. This was significant in that it removed the error sensor as well as the control actuator from the sound field and placed them both on the plate, thus allowing for control of a sound field while constraining the sensing and actuating to the structure.

With this idea in mind, sensing techniques for volume velocity were investigated. Two of the methods pursued were accelerometer arrays [6] and distributed piezoelectric films [7]. An accelerometer array can give an accurate estimate of the volume velocity of the plate. However, it requires a large number of sensors to be implemented. For example, on a plate with dimensions of $0.278 \mathrm{~m} \times 0.247 \mathrm{~m}$ it was predicted that 16 to 25 accelerometers would be needed to effectively measure the volume velocity [6]. With larger plates this number increases. Making a measurement in this manner does not reduce the complexity of the hardware required for control, thus eliminating one of the potential benefits of ASAC.

A distributed piezoelectric film avoids the necessity of a large number of error sensors to sense volume velocity, requiring only a single piezoelectric patch. In this case, the difficulty is not in the number of sensors required but in the fact that the sensor needs to be designed specifically for the geometry in question. For a plate of size $0.314 \mathrm{~m} \times 0.414 \mathrm{~m}$ a PVDF film was used with dimensions of $0.306 \mathrm{~m} \times 0.406 \mathrm{~m}$ [7]. For a different size plate an entirely different sensor would need to be designed and constructed. Thus, while effective, this method is highly geometry dependent and difficult to implement.

Other methods were developed for sensing radiation mode shapes, such as optimally shaped piezoelectric films designed to target the specific structural vibration modes contributing significantly to the important acoustic radiation modes [8]. This allowed for the first radiation mode to be targeted specifically as well as several other higher but significantly radiating modes. Structural energy methods have also been investigated but with limited success [9].

Optimally shaped piezoelectric films have the benefit of targeting the radiation modes of a structure more directly. Because only a few radiation modes contribute to sound radiation at low frequencies and only a few structural modes contribute significantly to each radiation mode, this method requires a smaller number of sensors than volume velocity. If the first radiation mode was the only mode of interest (also the target of volume velocity), 3 to 4 sensors would be required, depending on the frequency, to sense the contributing structural modes. By using 6 to 8 sensors, this method could also target multiple radiation modes [8].

However, similar to the distributed piezoelectric film, this approach is highly dependent on the geometry. In order to sense specific structural modes the piezoelectric sensors need to be shaped appropriately. Significant analysis of the structural and radiation modes is required to determine which modes are important and the required sensor shapes.

As previously described, many of the suggested methods of sensing for ASAC have had drawbacks due to the large number of sensors required or sensor's dependency on geometry. This paper applies a relatively new structural quantity known as WSSG to the control of clamped flat plates.

WSSG has been shown to be a nearly uniform structural quantity on vibrating simply supported rectangular plates [10, 11]. This uniformity allows for insensitivity to sensor location, reducing the analysis required in choosing an appropriate sensing location, a difficulty encountered when making other point measurements of vibration. The quantity itself, which is a sum of weighted spatial gradients, can be measured using a single, closely spaced array of four accelerometers and has been shown to provide better or similar control to other standard ASAC minimization quantities. WSSG has also been developed and found to be effective on ribbed plates, using a finite element, as opposed to analytical, model [12]. These developments demonstrated the effectiveness of WSSG. However, they did not thoroughly investigate or develop WSSG on clamped plates. The purpose of this paper is to develop the WSSG approach for a rectangular plate with clamped boundary conditions. This is desirable due to the greater practical applicability of clamped plates as compared to simply supported plates. It is also significant because it reveals some of the underlying physical mechanisms associated with the choice of weights for WSSG. These mechanisms were not discussed in the simply supported or ribbed plate papers.

\section{Development}

A thin vibrating plate is governed by the equation

$$
\begin{aligned}
& D\left(\frac{\partial^{4}}{\partial x^{4}}+2 \frac{\partial^{4}}{\partial x^{2} \partial y^{2}}+\frac{\partial^{4}}{\partial y^{4}}\right) w(x, y)-\bar{m} \omega^{2} w(x, y) \\
& \quad=F(x, y),
\end{aligned}
$$

where $D$ is the bending stiffness, $w$ is the displacement, $\omega$ is the excitation frequency, and $\bar{m}$ is the mass per unit area [13]. The plate excitation is assumed to be time harmonic; therefore the displacement and force expressions can be assumed to be multiplied by $e^{j \omega t}$ although not stated explicitly. This is an assumption made throughout the remainder of this paper. Also, for the current formulation the plate will be assumed to be excited by a series of point forces, so that $F(x, y)=$ $\sum_{q} F_{q} \delta\left(x-x_{q}\right) \delta\left(y-y_{q}\right)$, with $F_{q}$ indicating the $q$ th applied force as a complex number, $\delta(\cdot)$ indicating the Dirac delta function, and $x_{q}$ and $y_{q}$ indicating the location of the $q$ th force.

An exact analytical solution to (1) for clamped rectangular plates is not available but an approximate analytical solution can be developed by assuming that the clamped plate modes are the product of beam mode shapes [14]. Mathematically this solution is given as

$$
w(x, y)=\sum_{q} \sum_{m}^{\infty} \sum_{n}^{\infty} \frac{F_{q} \phi_{m}\left(x_{q}\right) \phi_{n}\left(y_{q}\right) \phi_{m}(x) \phi_{n}(y)}{D\left(I_{1} I_{2}+2 I_{3} I_{4}+I_{5} I_{6}\right)-\bar{m} \omega^{2} I_{2} I_{6}},
$$




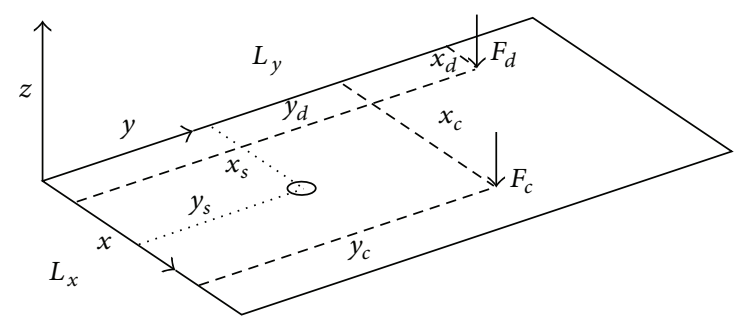

FIGURE 1: Coordinate system and force application on plate.

where

$$
\begin{gathered}
\phi_{p}(\xi)=C_{1}\left(\frac{\lambda_{p} \xi}{L_{\xi}}\right)-\frac{C_{1}\left(\lambda_{p}\right)}{S_{1}\left(\lambda_{p}\right)} S_{1}\left(\frac{\lambda_{p} \xi}{L_{\xi}}\right), \\
C_{1}(\xi)=\cosh (\xi)-\cos (\xi), \\
S_{1}(\xi)=\sinh (\xi)-\sin (\xi), \\
I_{1}=\int_{0}^{L_{x}} \phi_{m}^{(4)}(x) \phi_{m}(x) d x \quad I_{2}=\int_{0}^{L_{y}} \phi_{n}^{2}(y) d y, \\
I_{3}=\int_{0}^{L_{x}} \phi_{m}^{\prime \prime}(x) \phi_{m}(x) d x \quad I_{4}=\int_{0}^{L} \phi_{n}^{\prime \prime}(y) \phi_{n}(y) d y, \\
I_{5}=\int_{0}^{L_{y}} \phi_{n}^{(4)}(y) \phi_{n}(y) d y \quad I_{6}=\int_{0}^{L} \phi_{m}^{2}(x) d x .
\end{gathered}
$$

The subscripts $m$ and $n$ indicate structural mode numbers, and the values for $\lambda_{p}$ and the eigenvalues of the system are given by the characteristic equation

$$
\cosh \left(\lambda_{p}\right) \cos \left(\lambda_{p}\right)=1 .
$$

$\phi(\cdot)$ is the assumed shape function, and $\phi^{\prime \prime}(\cdot)$ and $\phi^{(4)}(\cdot)$ refer to the second and fourth spatial derivatives. The typical geometry and application of forces can be seen in Figure 1, where the subscripts $d, c$, and $s$ represent the disturbance force, control force, and sensor locations, respectively. Using the Rayleigh-Ritz method and comparing experimental tests to predictions made by this model, this method has been shown to predict the natural frequencies within an acceptable degree of error [15].

2.1. Derivation of the Objective Function for Clamped Plates. Previously, WSSG was defined [10] as

$$
\mathrm{WSSG}=\alpha(w)^{2}+\beta\left(\frac{\partial w}{\partial x}\right)^{2}+\gamma\left(\frac{\partial w}{\partial y}\right)^{2}+\delta\left(\frac{\partial^{2} w}{\partial x \partial y}\right)^{2},
$$

where the choice of weights $\alpha, \beta, \gamma$, and $\delta$ was important in deriving a uniform quantity for the simply supported rectangular plate.

While (6) is fundamentally the same with regard to control for both the time harmonic simply supported and clamped plates the choice of weights changes with the boundary conditions and is dependent on the eigenvalues of the solution to (1). To determine the weights, the derivatives of (2) with respect to $x$ and $y$, as shown by ((7)-(9)), are used:

$$
\begin{aligned}
& \frac{\partial w}{\partial x}=\sum_{q} \sum_{m}^{\infty} \sum_{n}^{\infty} \frac{F_{q} \phi_{m}\left(x_{q}\right) \phi_{n}\left(y_{q}\right) \phi_{m}^{\prime}(x) \phi_{n}(y)}{D\left(I_{1} I_{2}+2 I_{3} I_{4}+I_{5} I_{6}\right)-\bar{m} \omega^{2} I_{2} I_{6}}, \\
& \frac{\partial w}{\partial y}=\sum_{q} \sum_{m}^{\infty} \sum_{n}^{\infty} \frac{F_{q} \phi_{m}\left(x_{q}\right) \phi_{n}\left(y_{q}\right) \phi_{m}(x) \phi_{n}^{\prime}(y)}{D\left(I_{1} I_{2}+2 I_{3} I_{4}+I_{5} I_{6}\right)-\bar{m} \omega^{2} I_{2} I_{6}},
\end{aligned}
$$

$$
\frac{\partial^{2} w}{\partial x \partial y}=\sum_{q} \sum_{m}^{\infty} \sum_{n}^{\infty} \frac{F_{q} \phi_{m}\left(x_{q}\right) \phi_{n}\left(y_{q}\right) \phi_{m}^{\prime}(x) \phi_{n}^{\prime}(y)}{D\left(I_{1} I_{2}+2 I_{3} I_{4}+I_{5} I_{6}\right)-\bar{m} \omega^{2} I_{2} I_{6}},
$$

where

$$
\begin{gathered}
\phi_{m}^{\prime}(x)=\frac{\lambda_{m}}{L_{x}}\left[S_{2}\left(\frac{\lambda_{m} x}{L_{x}}\right)-\frac{C_{1}\left(\lambda_{m}\right)}{S_{1}\left(\lambda_{m}\right)} C_{1}\left(\frac{\lambda_{m} x}{L_{x}}\right)\right], \\
\phi_{n}^{\prime}(y)=\frac{\lambda_{n}}{L_{y}}\left[S_{2}\left(\frac{\lambda_{n} y}{L_{y}}\right)-\frac{C_{1}\left(\lambda_{n}\right)}{S_{1}\left(\lambda_{n}\right)} C_{1}\left(\frac{\lambda_{n} y}{L_{y}}\right)\right], \\
S_{2}(q)=\sinh (q)+\sin (q) .
\end{gathered}
$$

Each of the WSSG terms for the first mode can be seen in Figure 2. Note how the first term has maximum values in the middle, the first derivative terms have maximum values towards the outside edges, and the cross derivative term has maximum values near the corners. By summing these four terms with the weights chosen to normalize the maximum values, a nearly uniform field can be created.

Terms in (7) are scaled by a factor of $\lambda_{m} / L_{x}$ when the derivative is taken as shown by (10). Likewise, (8) and (9) are scaled by factors of $\lambda_{n} / L_{y}$ and $\lambda_{m} \lambda_{n} / L_{x} L_{y}$ as shown by (10) and (11). These scaling factors are responsible for the difference in magnitudes of the terms. To create a uniform WSSG field, the inverse of these terms was chosen and squared to form the weights. These inverse expressions, as the clamped plate weights, are given on the first line of Table 1. Note that each of the weights was scaled by $\alpha$. There is no specific value defined for $\alpha$, which can be used to scale all the terms by an equal amount. Normally the value of $\alpha$ is set to unity.

An alternative way to define the weights is in terms of the wave number. The wave numbers are inherent in the shape functions described by (3). For the clamped plate the wave number is $k=\lambda / L$, which can be substituted into the weights equations in the first line of Table 1 to yield the general weights expressions shown on the second line of Table 1. For the case of the simply supported plate $k_{m}=$ $m \pi / L_{x}$ which yields the correct weight expression [10] $\beta_{m}=$ $\left(L_{x} / m \pi\right)^{2}$. This can be demonstrated in a similar manner for $\gamma_{n}$ and $\delta_{m n}$, verifying that the general weight expressions are independent of boundary conditions and reduce down to the correct weights when the wave numbers, corresponding to the chosen boundary condition, are used. This general expression is significant because it shows that each term is weighted by the corresponding wave number components 
TABLE 1: Expressions for the weights in WSSG. The average weights shown were calculated using the geometry described in Table 2.

\begin{tabular}{llcc}
\hline Quantity & $\frac{\partial w}{\partial x}$ & $\frac{\partial w}{\partial y}$ & $\frac{\partial^{2} w}{\partial x \partial y}$ \\
\hline Clamped weights & $\beta_{m}=\alpha\left(\frac{L_{x}}{\lambda_{m}}\right)^{2}$ & $\gamma_{n}=\alpha\left(\frac{L_{y}}{\lambda_{n}}\right)^{2}$ & $\delta_{m n}=\alpha\left(\frac{L_{x} L_{y}}{\lambda_{m} \lambda_{n}}\right)^{2}$ \\
\hline General weights & $\beta_{m}=\alpha\left(\frac{1}{k_{m}}\right)^{2}$ & $\gamma_{n}=\alpha\left(\frac{1}{k_{n}}\right)^{2}$ & $\delta_{m n}=\alpha\left(\frac{1}{k_{m} k_{n}}\right)^{2}$ \\
\hline Average weights & $\beta_{\text {ave }}=0.00595$ & $\gamma_{\text {ave }}=0.00897$ & $\delta_{\text {ave }}=4.92 \times 10^{-5}$ \\
\hline
\end{tabular}

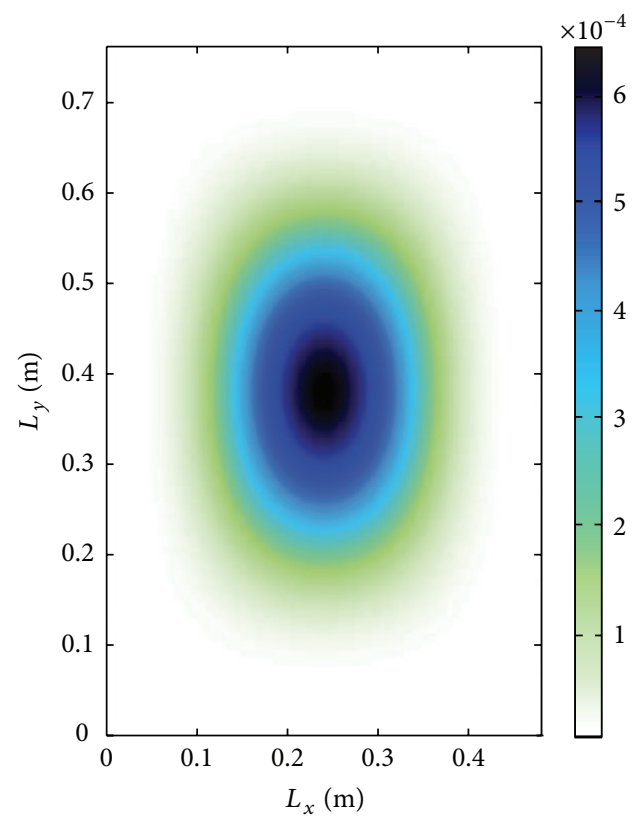

(a) $w^{2}$



(c) $(\partial w / \partial y)^{2}$



(b) $(\partial w / \partial x)^{2}$

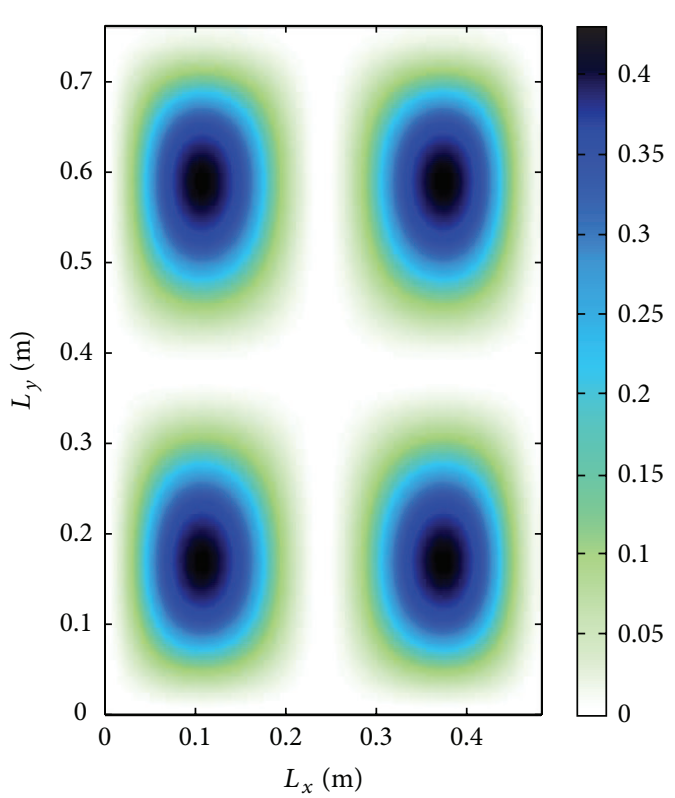

(d) $\left(\partial^{2} w / \partial x \partial y\right)^{2}$

FIgURe 2: Analytical WSSG terms for the first structural mode. 
TABLE 2: Plate properties and dimensions for control simulations.

\begin{tabular}{lc}
\hline Property & Value \\
\hline Length in $x, L_{x}$ & $0.483 \mathrm{~m}$ \\
Length in $y, L_{y}$ & $0.762 \mathrm{~m}$ \\
Thickness & $0.0031 \mathrm{~m}$ \\
Young's modulus & $69.8 \mathrm{GPa}$ \\
Poisson's ratio & 0.33 \\
Density & $2700 \mathrm{~kg} / \mathrm{m}^{3}$ \\
Damping ratio & $2 \%$ \\
\hline
\end{tabular}

TABLE 3: The first fifteen natural frequencies and mode shapes for the plate described in Table 2.

\begin{tabular}{lllc}
\hline Mode & $m$ & $n$ & Natural frequency $(\mathrm{Hz})$ \\
\hline 1 & 1 & 1 & 86 \\
2 & 1 & 2 & 128 \\
3 & 1 & 3 & 204 \\
4 & 2 & 1 & 214 \\
5 & 2 & 2 & 253 \\
6 & 1 & 4 & 299 \\
7 & 2 & 3 & 327 \\
8 & 2 & 4 & 406 \\
9 & 3 & 1 & 410 \\
10 & 1 & 5 & 430 \\
11 & 3 & 2 & 452 \\
12 & 3 & 3 & 531 \\
13 & 2 & 5 & 538 \\
14 & 1 & 6 & 583 \\
15 & 3 & 4 & 599 \\
\hline
\end{tabular}

and allows for the selection of weights on plates with nonideal shapes if the wave number components in $x$ and $y$ can be determined.

At this point, the derived weights are only valid for individual mode numbers $m$ and $n$, while the total displacement given in (2) is a sum over an infinite number of modes. While individual modes can be weighted analytically, this cannot be done experimentally, as the contributions to displacement from each of the modes cannot be separated in real time. Several different weighting schemes have been investigated for the non-mode-specific weights with very similar results in simulated control. It can be concluded that the minimization quantity is not overly sensitive to the general weighting scheme as long as the weights are of the appropriate order of magnitude. The simplest method used to compute results that provided excellent results was averaging the $\beta_{m}, \gamma_{n}$, and $\delta_{m n}$ values over the first 15 modes of the geometry examined.

2.2. Uniformity of WSSG Field. With the weights chosen as described above, the uniformity of the WSSG field on the clamped plate can be demonstrated. Using the equations shown in Table 1 and the plate geometry and material properties defined in Table 2 the natural frequencies and mode shapes were computed. These are given in Table 3 . The WSSG

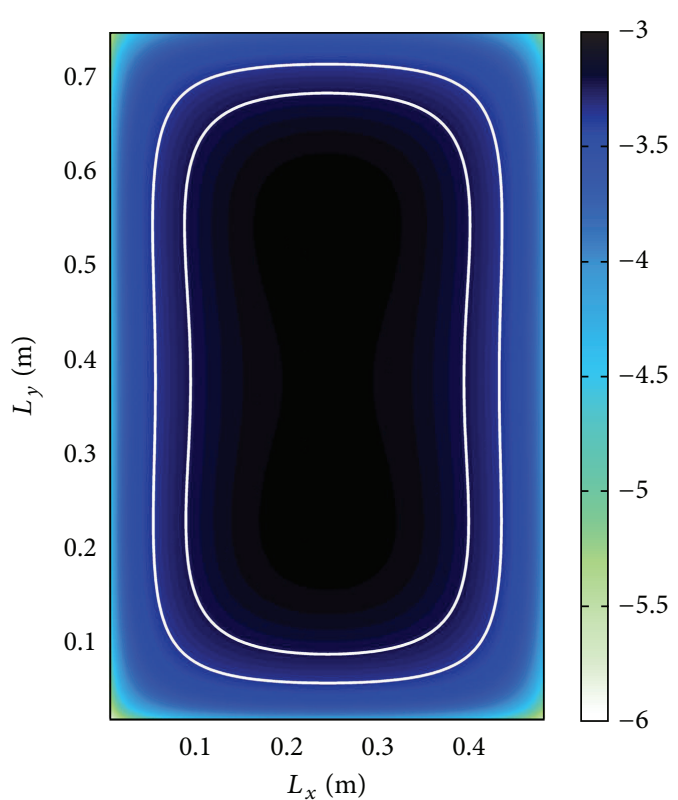

FIGURE 3: WSSG field for the clamped plate 1, 2 mode in $\mathrm{dB}$, relative to the maximum value on the plate, at the 1,2 mode natural frequency. The inner white line represents the $3 \mathrm{~dB}$ down contour and the outer one represents the $6 \mathrm{~dB}$ down.

field for the second mode, $(1,2)$, is found by exciting the plate at the second mode resonance frequency and calculating the weights and summing over only the second mode in (2), (7), (8), and (9). This field is shown in Figure 3, in decibels relative to the maximum value on the plate, to demonstrate uniformity. The inner white contour represents the boundary of the $3 \mathrm{~dB}$ down region and contains approximately $50 \%$ of the plate. The outer white contour represents the boundary of the $6 \mathrm{~dB}$ down region and contains approximately $66 \%$ of the plate. The standard deviation of the WSSG field is $6.7 \mathrm{~dB}$. The simply supported plate, used in previous work [10], was found to be uniform to within $\pm 0.01 \%$ at the second mode. The clamped boundary conditions, and therefore the introduction of hyperbolic sines and cosines in the shape functions, are the cause of this difference in uniformity.

A more practical case occurs when WSSG is summed over multiple modes (in (2), (7), (8), and (9)), more closely modeling the displacement of an actual plate when it is excited. In this case the average weight values shown in Table 1 are used. The WSSG field summed over the first 15 modes, given in Table 3, using the average weights, is shown in Figure 4. In this case the $3 \mathrm{~dB}$ down region contains approximately $31 \%$ of the plate, and the $6 \mathrm{~dB}$ down region contains approximately $50 \%$ of the plate, so with more modes included the WSSG field becomes slightly less uniform. The standard deviation of the WSSG field in this case is $7.2 \mathrm{~dB}$, which is $0.5 \mathrm{~dB}$ higher than for a single mode. For the simply supported plate in this situation, using average weights, excited at the second mode natural frequency, the $3 \mathrm{~dB}$ down region contained about $80 \%$ of the plate, and the entire plate was within $4 \mathrm{~dB}$, once again 


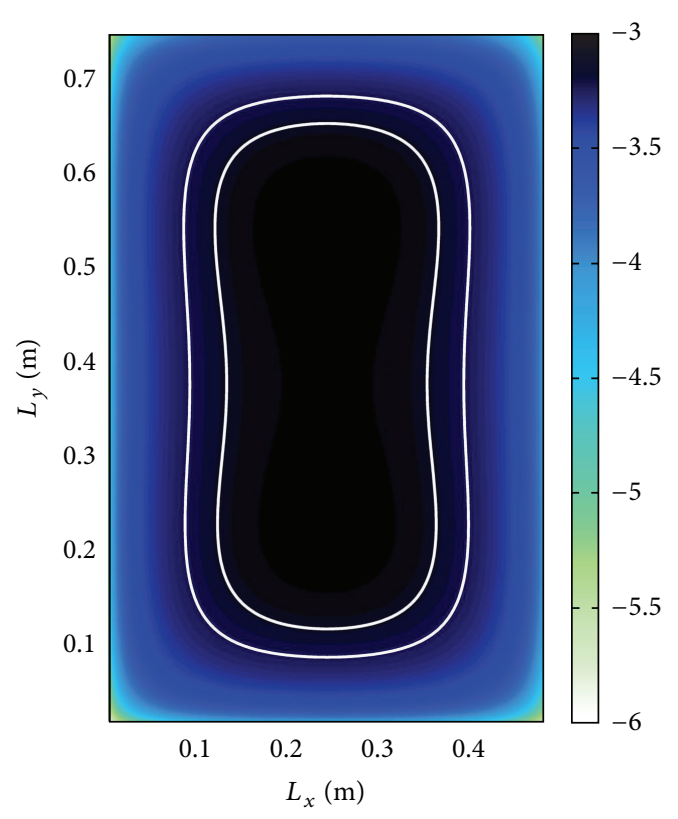

FIGURE 4: WSSG field summed over the first 15 modes, at the 1, 2 mode natural frequency, in $\mathrm{dB}$ relative to the maximum value on the plate. The inner white line represents the $3 \mathrm{~dB}$ down contour and the outer one represents the $6 \mathrm{~dB}$ down.

more uniform due to the difference in boundary conditions and the resulting shape functions.

When WSSG is summed over multiple modes the uniformity decreases due to the contributions from other modes to the displacement of the plate. Also, the WSSG field on the clamped plate is less uniform than the WSSG field on the simply supported plate [10] due to the difference in boundary conditions. The clamped boundary prevents the plate from rotating at the edges, therefore driving the derivative terms to zero at the boundaries of the plate, which can be seen in Figures 3 and 4. At higher modes and frequencies this transition area decreases. These results suggest that sensors should be placed away from the clamped edges of a plate. Another important point to note is that while Figures 3 and 4 show the WSSG field at the second mode, there are no nodal lines in the field. In general, nodal lines in the WSSG field do not appear regardless of the mode or frequency of excitation. This property significantly simplifies the choice of sensor location, reducing the importance of the exact location.

2.3. Comparison to Radiation Modes. One of the interesting properties noted in the original development of WSSG [10] was the relationship between the radiation mode shapes and the shape of the spatial derivatives. The radiation modes of a plate are derived from the radiation resistance matrix. The radiation resistance matrix discretizes the plate geometry and provides a simplified and computationally more efficient method for calculating the radiated sound power at low frequencies. It is derived by dividing the plate into elemental radiators and calculating the mutual radiation impedances between each elemental radiator and all others. This matrix is given, for a rectangular plate [4], by

$$
\begin{aligned}
\mathbf{R}= & \frac{\omega^{2} \rho_{0} A_{e}^{2}}{4 \pi c} \\
& {\left[\begin{array}{cccc}
1 & \frac{\sin \left(k R_{12}\right)}{k R_{12}} & \cdots & \frac{\sin \left(k R_{1 N}\right)}{k R_{1 N}} \\
\frac{\sin \left(k R_{21}\right)}{k R_{21}} & 1 & & \vdots \\
\vdots & & \ddots & \\
\frac{\sin \left(k R_{N 1}\right)}{k R_{N 1}} & \cdots & & 1
\end{array}\right], }
\end{aligned}
$$

where $\rho_{0}$ is the density of the surrounding fluid, $A_{e}$ is the elemental area, $c$ is the speed of sound in the fluid, $R_{i j}$ is the distance from the $i$ th to the $j$ th element, $N$ is the total number of elements, and the other terms are as previously defined. Using the radiation resistance matrix, the sound power is calculated as

$$
P=\mathbf{v}_{e}^{H} \mathbf{R} \mathbf{v}_{e}
$$

where $\mathbf{v}_{e}$ is a vector containing the velocity of each element and $H$ signifies the Hermitian transpose [6].

The radiation mode shapes are found as the eigenvectors of the radiation resistance matrix and break the matrix up into a set of mutually orthogonal vectors which reveal the underlying radiation mechanisms of the structure. The radiation mode shapes, which are functions of geometry and frequency, but not boundary conditions, generally only have a few structural modes which contribute significantly to them. Thus, by targeting the radiation modes, the relevant radiating structural modes are also targeted. At low frequencies, it has also been found that only a few of the radiation modes have any significant contribution to sound radiation [4].

The first four radiation modes of the rectangular plate are shown in Figure 5 and the four terms of WSSG are shown in Figure 2. Note the similarity between these four terms. The first radiation mode is similar in shape to the displacement, $w$, which is comparable to a monopole source; the second and third radiation modes are similar to the first derivatives with respect to $x$ and $y$ and can be compared to dipole like radiation; the fourth radiation mode is similar to the cross derivative and is similar to quadrupole like radiation. Although these comparisons are made loosely and are not exact on the first mode of the clamped plate, at more complicated structural modes the derivative terms locally closely resemble the radiation mode shapes. It is hypothesized that this similarity is connected with the observed behavior that allows for a single or local measurement on the plate to control the radiation mode shapes globally, as opposed to volume velocity, which required a global measurement of the plate to control the first radiation mode. These similarities, which were also found for the simply supported plate, suggest that WSSG will be effective at targeting the first four radiation mode shapes for a clamped plate and will therefore be a good candidate for use in ASAC. 


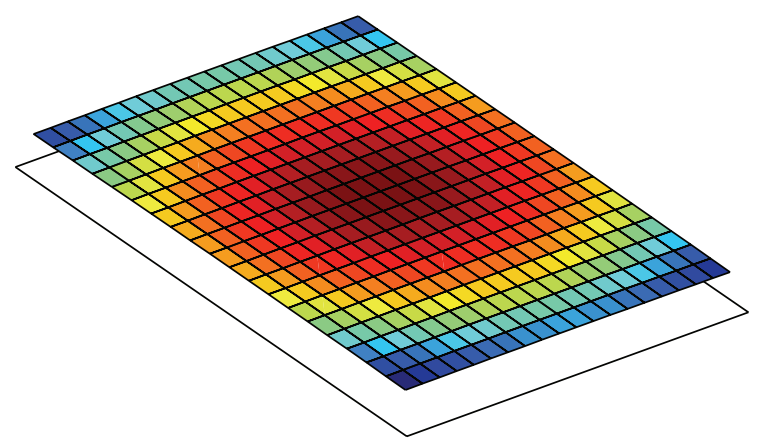

(a) Mode 1

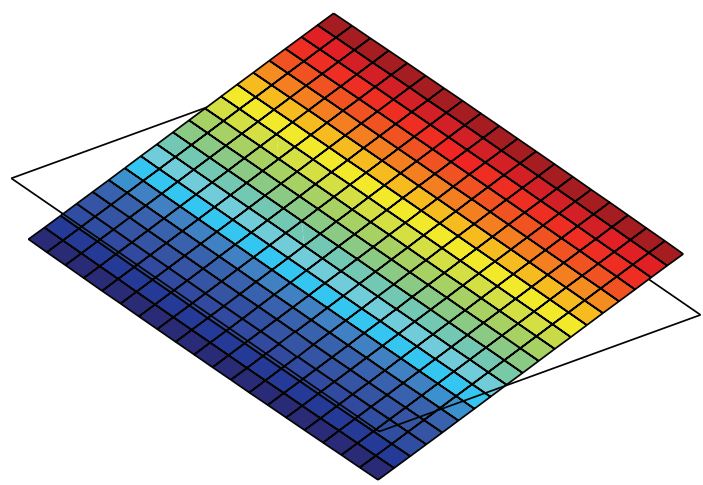

(c) Mode 3

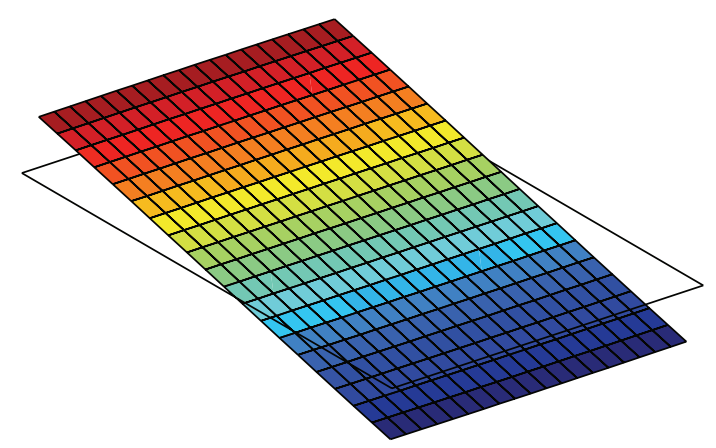

(b) Mode 2

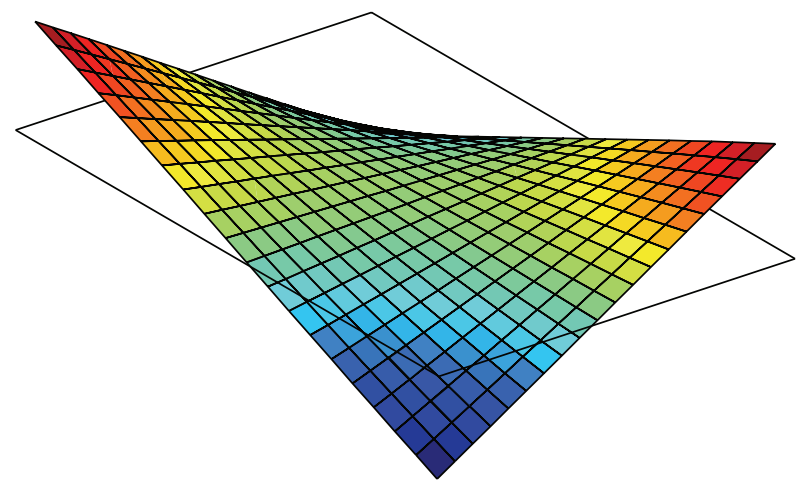

(d) Mode 4

FIGURE 5: The first four radiation mode shapes for a rectangular plate.

Because of the way in which the weights are calculated, a uniform WSSG field is created. The uniformity of the field is considered an advantage as far as sensitivity to sensor placement is concerned. However it can be viewed in light of weighting the radiation modes. By weighting each of the terms the same, each of the radiation modes is given the same weight in the control algorithm, thus targeting each of the first four radiation modes equally.

\section{Numerical Simulations}

To determine the effectiveness of WSSG control on a clamped plate, a numerical simulation was developed. The plate dimensions and material properties for the simulation are given in Table 2. Equations ((2) and (6)-(12)) were used as the analytical model for the plate. As stated previously, point forces were used to excite and control the plate. The locations of the disturbance and control forces, as well as the sensor location, are given in Table 4. The control was simulated by finding the optimal control force for each frequency to minimize WSSG at the sensor location. The radiated sound power was then calculated by using elementary radiators as described in (14).

In order to demonstrate the effectiveness of WSSG it was compared to results achieved from minimizing volume velocity. For the analytical simulation, volume velocity was found by discretizing the plate into 60 elements and estimating the velocity across each individual element as the velocity at
TABLE 4: Locations for the disturbance, control, and sensor locations of the simulations and experiments.

\begin{tabular}{lc}
\hline Quantity & Value \\
\hline$x_{d}$ & $0.083 \mathrm{~m}$ \\
$y_{d}$ & $0.629 \mathrm{~m}$ \\
$x_{c}$ & $0.083 \mathrm{~m}$ \\
$y_{c}$ & $0.127 \mathrm{~m}$ \\
$x_{s}$ & $0.286 \mathrm{~m}$ \\
$y_{s}$ & $0.432 \mathrm{~m}$ \\
\hline$d, c$, and $s$ represent disturbance, control, and sensor, respectively.
\end{tabular}

the center of the element. This number of elements was calculated as sufficient to give an accurate measure of volume velocity based on the methods described by Sors and Elliott [6].

For the simulated control, shown in Figure 6, the overall sound attenuation over the frequency range from 0 to $630 \mathrm{~Hz}$ is $28.8 \mathrm{~dB}$ with WSSG as the minimization quantity and $9.0 \mathrm{~dB}$ with volume velocity as the minimization quantity. WSSG consistently provides better control than volume velocity over the frequency spectrum for the case examined except in the range from about 180 to $210 \mathrm{~Hz}$.

The simulated control at each of the natural frequencies is shown in Figure 7. It can be easily seen from this that at most of the resonance frequencies (resonances 2, 4-8, 11, 13 , and 14) WSSG outperforms volume velocity. The success 


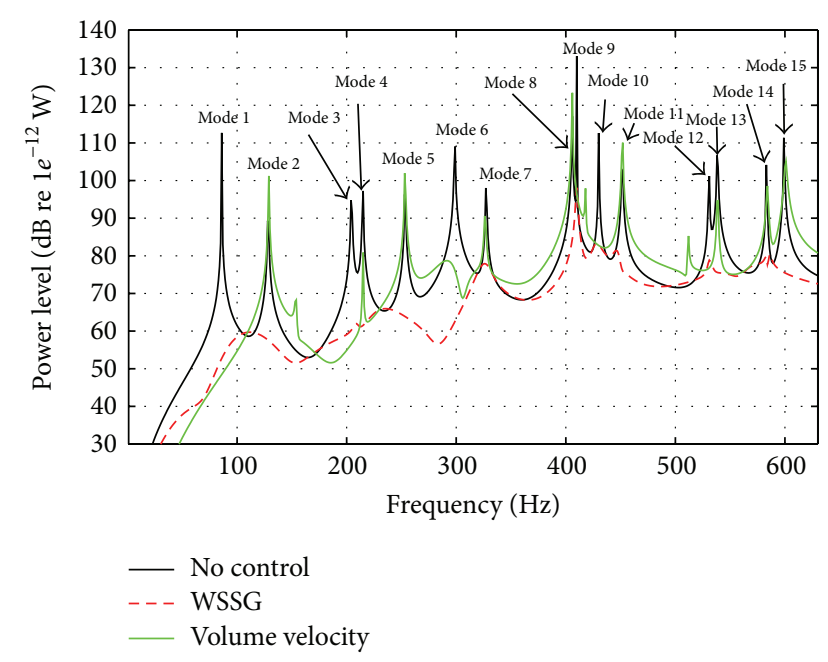

FIGURE 6: Simulated control of the clamped plate using WSSG and volume velocity as objective functions.

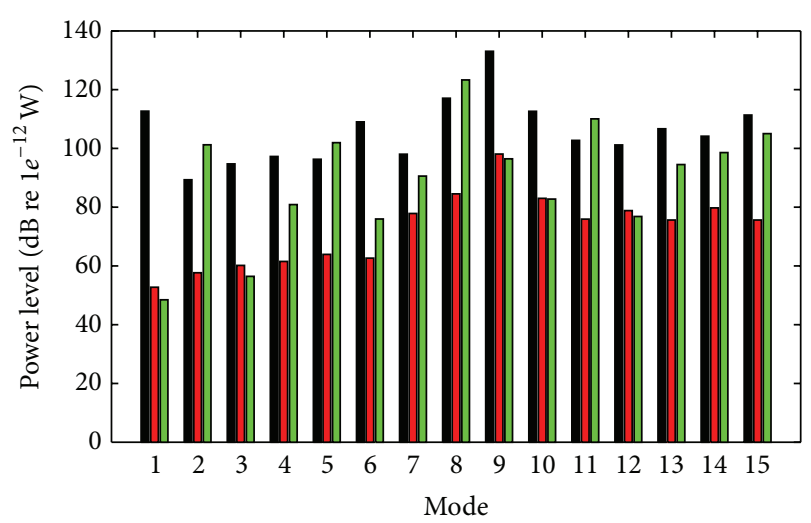

FIGURE 7: Simulated control sound power levels at the structural mode natural frequencies. The left column is the radiated sound level without control, the middle column represents the radiated sound level with control from WSSG, and the right column represents the radiated sound level with control from volume velocity.

of WSSG, especially at higher modes, is due to its ability to target the first four radiation modes as opposed to just the first radiation mode for volume velocity. The radiated sound power from each of the first four radiation mode shapes is shown in Figures 8-11. The overall sound attenuation for each of these modes is given in Table 5. When compared to volume velocity, WSSG provides improved control for all four modes, although both quantities provide appreciable control of the first mode. WSSG significantly outperforms volume velocity on the other three modes as expected from the theoretical development of the minimization quantity.

There are several things to note in Figures 8-11. Each of the radiation modes has a different set of structural modes contributing to their sound radiation. For example, in Figure 8 the first, third, and ninth structural modes radiate strongly, but the second, fourth, and fifth do not. In the case of the fifth structural mode there is no apparent contribution to the sound radiation of the first radiation mode. Modes that

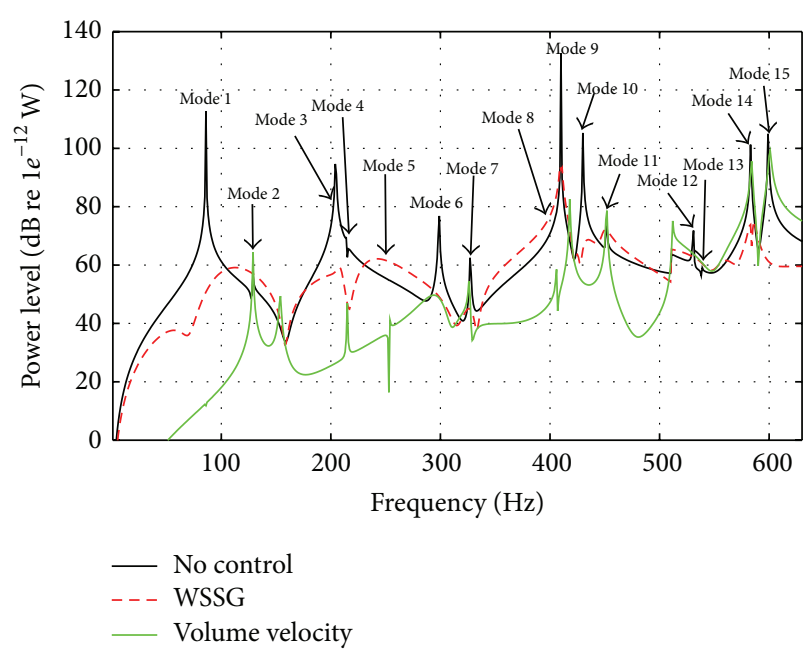

FIGURE 8: Sound radiated by the first radiation mode on the clamped rectangular plate.

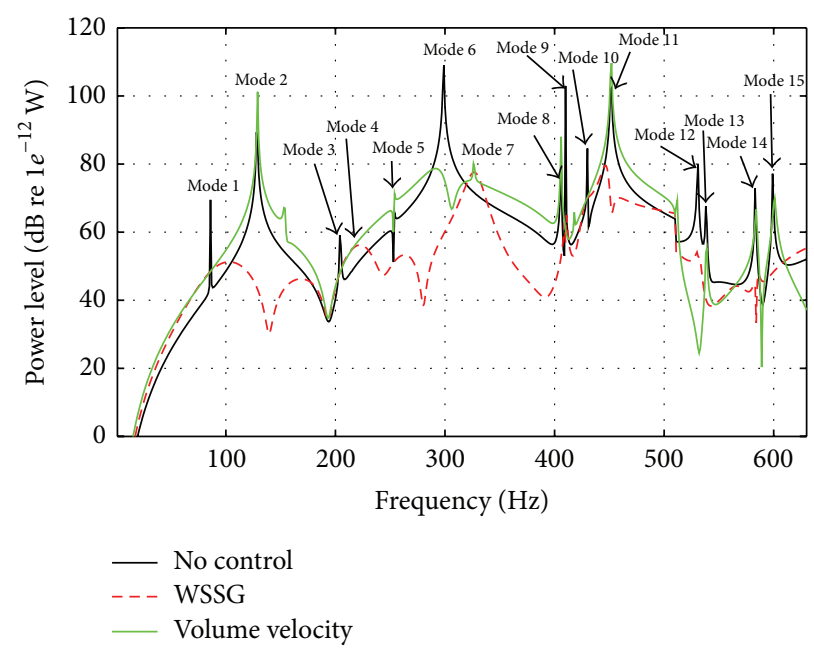

FIGURE 9: Sound radiated by the second radiation mode on the clamped rectangular plate.

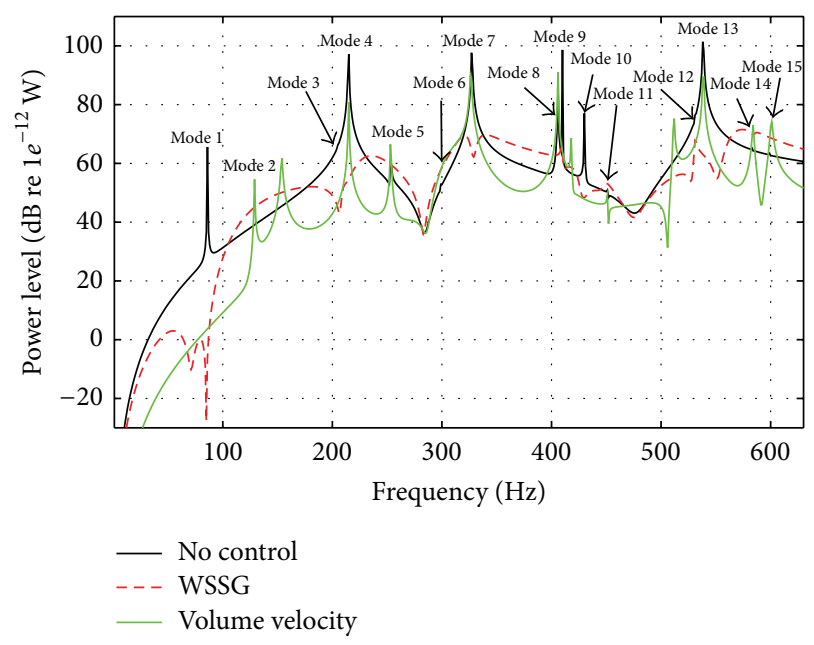

FIGURE 10: Sound radiated by the third radiation mode on the clamped rectangular plate. 


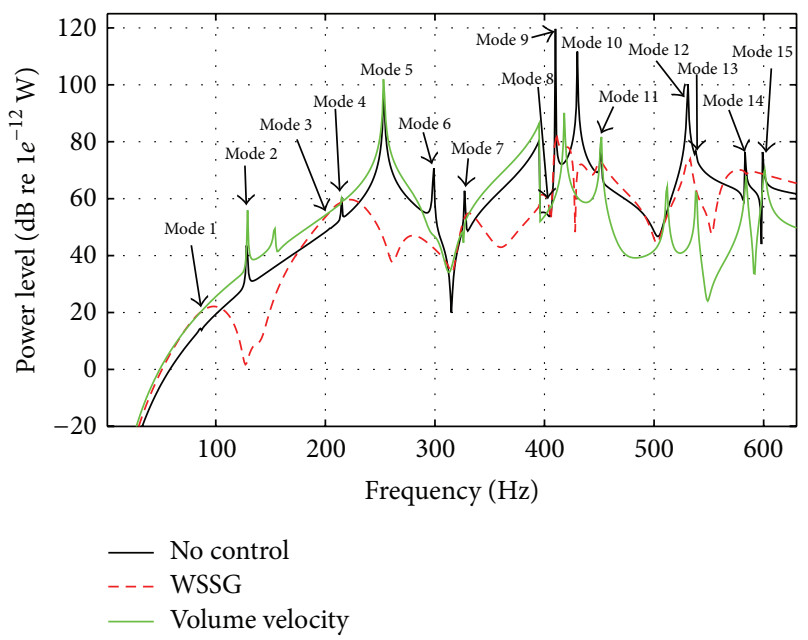

FIGURE 11: Sound radiated by the fourth radiation mode on the clamped rectangular plate.

TABLE 5: Overall sound attenuation in each of the four radiation modes targeted by WSSG, as shown in Figures 6-11.

\begin{tabular}{lcc}
\hline Mode & WSSG & Vol. vel. \\
\hline 1 & 32.1 & 27 \\
2 & 19.3 & -0.2 \\
3 & 16.0 & 9.0 \\
4 & 26.6 & 16 \\
Overall & 28.8 & 9.0 \\
\hline
\end{tabular}

do not appear to radiate sound power were still marked on the figures.

For the first radiation mode, volume velocity provides better or comparable control to WSSG over the frequency range, except for the 14th and 15th structural modes. The poor control of volume velocity and the good control of WSSG at these two modes caused WSSG to provide a better overall sound attenuation for the first radiation mode. For the other three radiation modes examined, WSSG outperformed volume velocity because it provides comparable or improved control at the significantly radiating natural frequencies. Specifically, in Figure 6 volume velocity does not provide significant control at modes $2,5,7,8,11,13,14$, or 15 . With the exception of modes 14 and 15 , which noticeably contribute to the radiated sound of multiple radiation modes, these structural modes contribute radiated sound through either the second, third, or fourth radiation mode, modes which volume velocity was not designed to target. Minimization of volume velocity is therefore unable to provide consistent control at these structural mode natural frequencies. These results show that WSSG does successfully target the first four radiation modes, allowing WSSG to provide a better overall sound attenuation than volume velocity.

Control of the plate with the sensor placed at random locations was also simulated to determine control sensitivity to sensor location. Five locations, given in Table 6, were chosen at random on the plate, and then control was simulated at
TABLE 6: The sensor locations used to test the sensitivity of WSSG to sensor placement on the plate of dimensions $0.483 \mathrm{~m} \times 0.762 \mathrm{~m}$.

\begin{tabular}{lccc}
\hline Sensor & $x(\mathrm{~m})$ & $y(\mathrm{~m})$ & Overall sound atten. $(\mathrm{dB})$ \\
\hline Default & 0.286 & 0.432 & 28.8 \\
1 & 0.088 & 0.566 & 25.4 \\
2 & 0.193 & 0.299 & 28.6 \\
3 & 0.126 & 0.499 & 27.9 \\
4 & 0.386 & 0.130 & 26.1 \\
5 & 0.208 & 0.538 & 28.0 \\
\hline
\end{tabular}

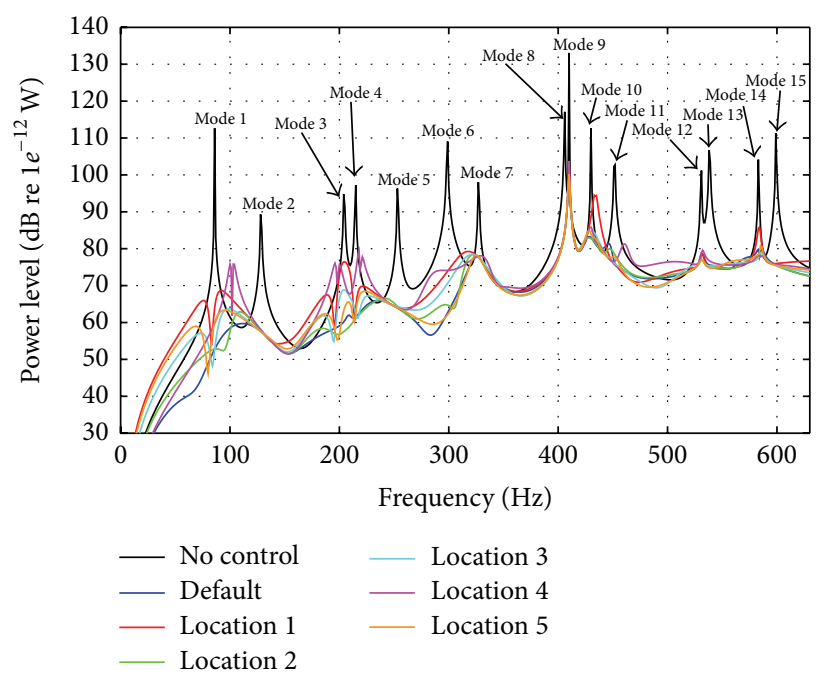

FIGURE 12: Simulated control of the clamped plate at multiple sensor locations.

those locations, along with the original location. The overall attenuation for each sensor location is shown in Table 6. A plot showing the control at these various locations is given in Figure 12. Each of the structural modes is successfully controlled for radiated sound power, although the ability to control mode 9 is reduced when compared to the other modes. This is due to the choice of control force location for these simulations.

Note the similarity in control regardless of the sensor location. The mean of the overall sound attenuation at these sensor locations is $27.5 \mathrm{~dB}$ with a standard deviation of $1.4 \mathrm{~dB}$, showing a high degree of consistency and thus verifying that the uniformity of the WSSG field, as discussed in Section 2.2, does result in control which is relatively insensitive to sensor location.

\section{Experimental Validation}

A clamped plate, made of 6061-T6 rolled aluminum, which had the same material properties and dimensions as the plate used in simulations (see Table 2), was used for the experimental validation. The clamped boundary condition was created by placing the plate between two stiff frames and bolting the frames together. A picture of the plate is shown in Figure 13. Spatially dense velocity measurements 


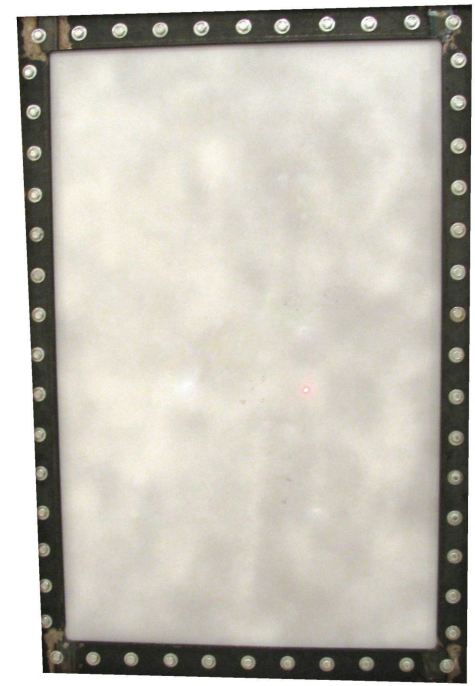

FIGURE 13: The experimental clamped-clamped plate.

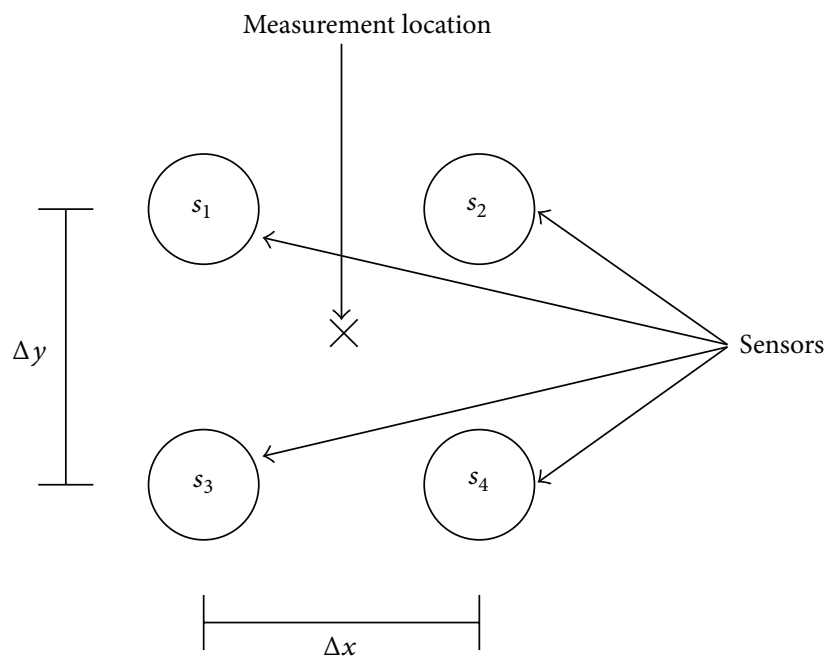

FIGURE 14: Schematic of accelerometer positions for WSSG measurement experimentally.

across the entire plate were made with a scanning laser Doppler vibrometer (SLDV) to ensure that a purely clamped boundary condition was closely approximated. To make these measurements, the plate was excited using a lightweight shaker attached to its corner. The SLDV data was used to estimate the natural frequencies of the system. These measured natural frequencies compared very closely to the theoretical clamped plate natural frequency predictions, confirming that the experimental setup satisfactorily approximated the ideal mathematical clamped boundary condition.

The plate was excited using shakers, suspended by bungee cords. The shakers were attached to the plate via stingers, which were glued in place. The WSSG quantity was measured at a point using a rectangular array of four accelerometers spaced $0.0254 \mathrm{~m}$ apart as shown in Figure 14. These were attached to the front of the plate.

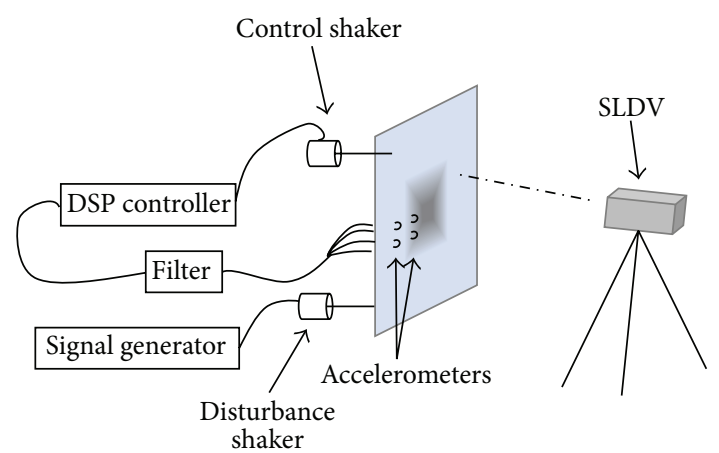

FIGURE 15: Schematic of the experimental setup.

Each of the terms in (6) was found numerically by calculating finite difference derivatives from the accelerometer signals at each sampled time step. The equations used to calculate each term were

$$
\begin{aligned}
w & \approx \frac{s_{1}+s_{2}+s_{3}+s_{4}}{4}, \\
\frac{\partial w}{\partial x} & \approx \frac{s_{2}-s_{1}+s_{4}-s_{3}}{2 \Delta x}, \\
\frac{\partial w}{\partial y} & \approx \frac{s_{1}-s_{3}+s_{2}-s_{4}}{2 \Delta y}, \\
\frac{\partial^{2} w}{\partial x \partial y} & \approx \frac{s_{2}-s_{1}+s_{3}-s_{4}}{\Delta x \Delta y},
\end{aligned}
$$

where $s_{i}$ represents the instantaneous acceleration measured by each accelerometer and $\Delta x$ and $\Delta y$ represent the $x$ and $y$ distance between the accelerometers, respectively, as shown in Figure 14. Because time harmonic excitation is used, the acceleration differs from the displacement only by a multiplicative constant. Thus, accelerations are used interchangeably with displacements here, with no impact on the result for minimizing WSSG.

Each term was calculated in the control algorithm software. A filtered-x LMS [16] algorithm was used to find the optimal phase and amplitude signal. This signal caused the control shaker to minimize the WSSG quantity at the chosen point. The sound power was calculated using the same method as that used in the numerical simulations, with the velocity of each elementary radiator being provided by the SLDV. Figure 15 shows a schematic of the experimental setup. The plate was placed in a window between two reverberation chambers. This provided a baffle between the two sides of the plate and isolated the plate from outside vibrations or noise, allowing for accurate sound measurements.

4.1. Experimental Validation of WSSG as a Uniform Metric on the Plate. The theoretical development of WSSG as a uniform parameter was validated by scanning the vibrating clamped plate with the SLDV at the natural frequencies. This was done with a small LDS V203 shaker to avoid mass loading the plate with the heavy shakers used in the control experiments. The individual terms of WSSG were calculated using (15) 


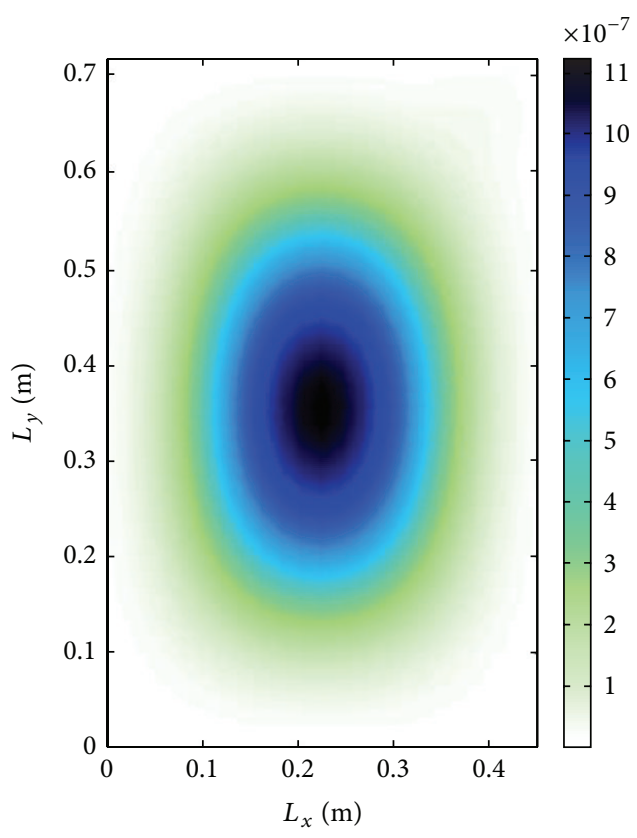

(a) $w^{2}$



(c) $(\partial w / \partial y)^{2}$

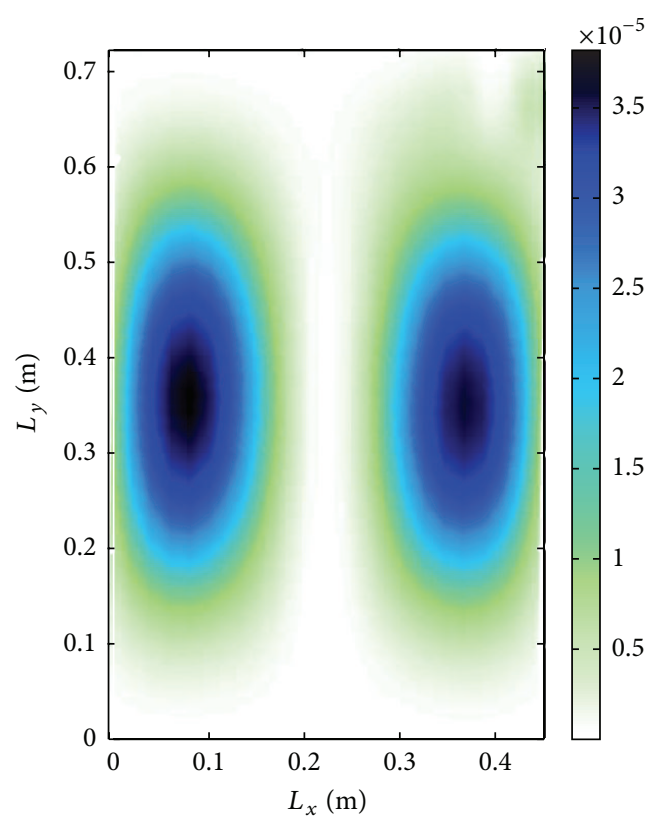

(b) $(\partial w / \partial x)$
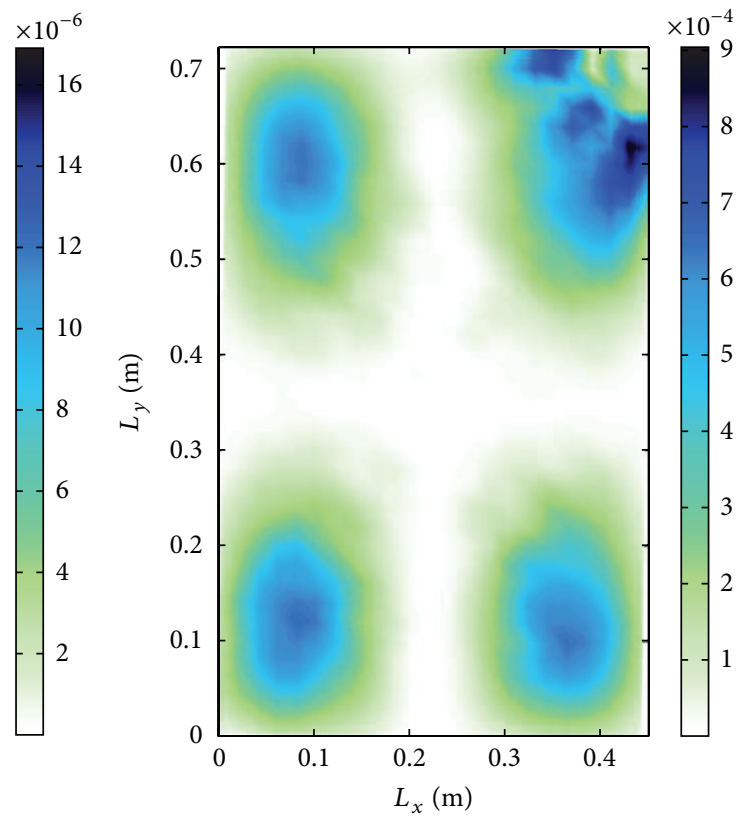

(d) $\left(\partial^{2} w / \partial x \partial y\right)^{2}$

FIGURE 16: Experimental WSSG terms for the first structural mode.

for each of the modes. The four WSSG terms with the plate excited at the first mode resonance frequency are shown in Figure 16. Each of the four terms closely matches with what the analytical expressions predicted in Figure 2, with the exception of some distortion in the upper right corner, where the shaker was located. Placing the shaker in the corner skewed the structural mode in the direction of the shaker. This caused distortions in the WSSG field which are most visible in Figure 16(d), the fourth term, because of its second order finite difference derivative. The second and third WSSG terms exhibit less distortion because they are only first order derivatives. For the second order derivative, due to this noise, a couple of outliers were removed so that the field could be seen more clearly.

When the four terms are summed as in (6), using the mode-specific weights for mode 1 shown on the first line of Table 1, a relatively uniform quantity over the plate is obtained as shown in Figure 17. A higher degree of uniformity is prevented because the clamped boundaries prevent any rotation and therefore force all derivative terms to zero at the edges. 


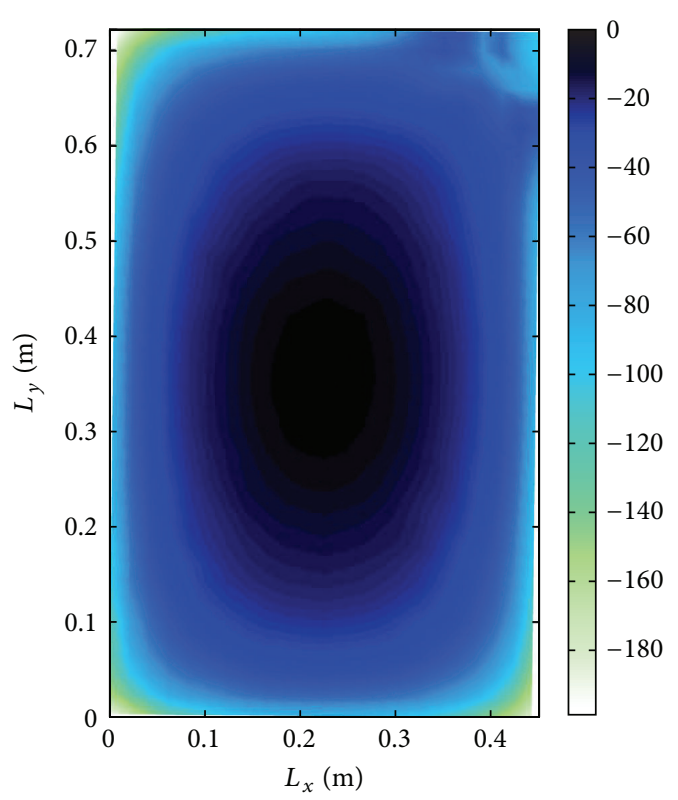

FIgURE 17: Total WSSG field for the plate excited at the first mode natural frequency, in $\mathrm{dB}$, with the first mode weights (first line of Table 1).

Figure 18 shows the WSSG fields for the plate excited at the resonance frequencies for modes $2-5$ with the ideal modespecific weights, given on the first line of Table 1, used at each mode. The second mode (Figure 18(a)) shows that the experimentally measured WSSG field qualitatively matches the simulated WSSG field shown in Figure 3 and further confirms the correctness of the theoretical development of WSSG.

The experimental results show that the WSSG field is not as uniform as the theory predicted. The lack of uniformity in Figure 18 can be traced to the nonuniform amplitudes of the antinodes in the displacement field. The simulations predicted that each antinode would have uniform amplitude, but this was not always the case in the experimental data. For example, on the second mode (the $(1,2)$ mode), the shaker was located in the lower right corner of the plate, much closer to the lower antinode than the upper. The SLDV scans showed that this caused the lower antinode to have a slightly higher amplitude than the upper antinode, which makes it impossible for the same WSSG values to be measured in the upper and lower regions of the plate at this frequency. However, the amplitudes are close enough in value for the $(1,2)$ mode, that placing the WSSG sensor in either section will still provide control of the plate. This was not the case for some of the other modes, where higher amplitude differences were measured between the antinodes.

Some of the higher modes had larger variations in the antinode amplitudes because of overlap between natural resonance frequencies. At closely spaced resonance frequencies the corresponding mode shapes often superimpose on top of each other, causing distortions in the mode shapes. This superimposition can cause significant differences in the antinode amplitudes, which causes WSSG to lose some of its uniformity. Thus, whenever a natural frequency was isolated (far away from any other natural frequency) a more uniform WSSG field was calculated, but when natural frequencies were close to each other, the WSSG field was less uniform. Some of these nonuniform effects were predicted by the computer simulations, but the experimental results showed even more pronounced amplitude differences.

4.2. Experimental Control Validation. The plate was set up as shown in Figure 15 with the disturbance and control shakers, as well as the WSSG sensor, in the same locations as in the simulations, given in Table 4. The results of sound power measurements, taken before and after control, are shown in Figure 19. Due to the time intensive nature of the experiments data was taken to verify the prediction of the simulations at or near the natural frequencies, and at small number of off resonance values, within a limited range of the frequency. This was deemed sufficient to validate the numerical simulations experimentally.

Figure 19, which shows the experimentally measured control over the frequency range from 60 to $370 \mathrm{~Hz}$, shows that the actual measured control is similar to that predicted by the simulations, shown in Figure 6, for the same frequency range. Several differences between Figures 6 and 19 are apparent which present some challenges in comparing the two tests. The experimental plate natural frequencies were generally lower than those predicted; however this is consistent with mass loading from the shakers. The shakers used for experimental validation and control of the plate were much heavier than the shaker used for the excitation and measurement of the natural frequencies, mass loading the plate in the control scenario. An example of this can be seen by looking at the fourth mode in the experiments, which was measured as the $(2,2)$ mode. In simulations the $(2,2)$ mode appears at $253 \mathrm{~Hz}$ but is shifted down to $231 \mathrm{~Hz}$ in the experimental control results.

A second difference between Figures 6 and 19 is that the experimental results are missing several of the resonance frequencies predicted in the simulations. Two probable reasons for these resonance frequencies to be missing are as follows: the experimental sampling resolution may not have been high enough to differentiate between two closely spaced natural frequencies, and the added mass and stiffness from the shakers may have distorted the structural modes in such a way that they were no longer a recognizable mode shape. It is possible that both reasons have a role in the missing natural frequencies.

For example, in Figure 19 the third natural frequency, at $200 \mathrm{~Hz}$, was shown in the SLDV scans to be an easily recognizable $(1,3)$ mode shape. However the $(1,3)$ mode shape was not perfect, and it appeared that a very weak (2, 1) mode might have been contributing to the response at this frequency, causing slight distortions to the $(1,3)$ mode. The $(2,1)$ mode, predicted as the fourth mode in simulations, had a natural frequency relatively close to the $(1,3)$ mode. The $(2,1)$ mode may have had a small enough amplitude in the experiments that it did not show up as a significant independent structural mode in the broadband SLDV scans, 


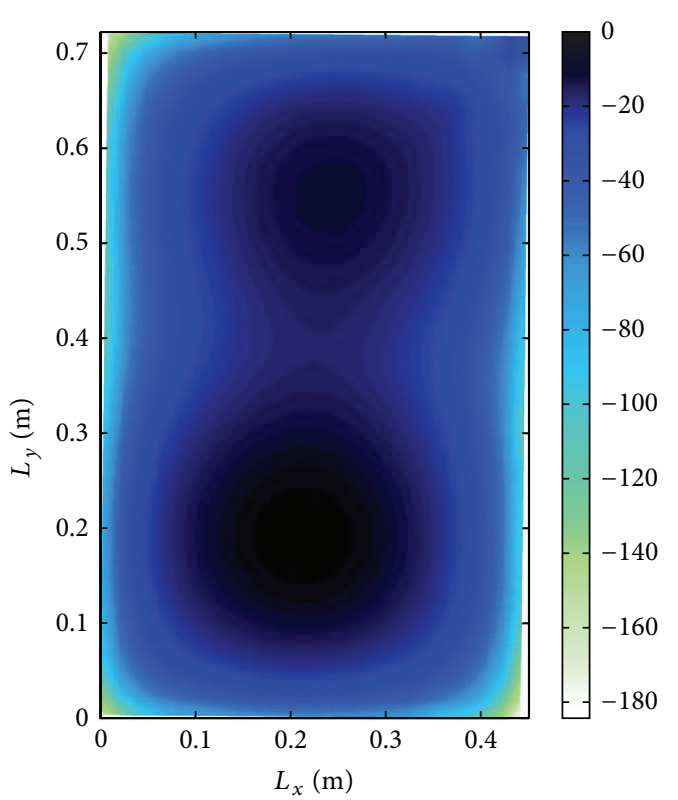

(a) Mode 2

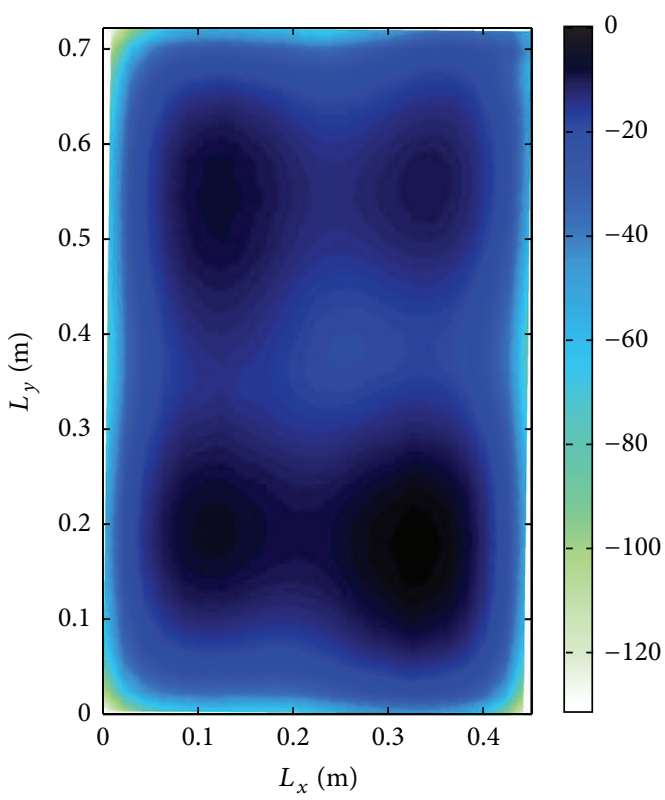

(c) Mode 4

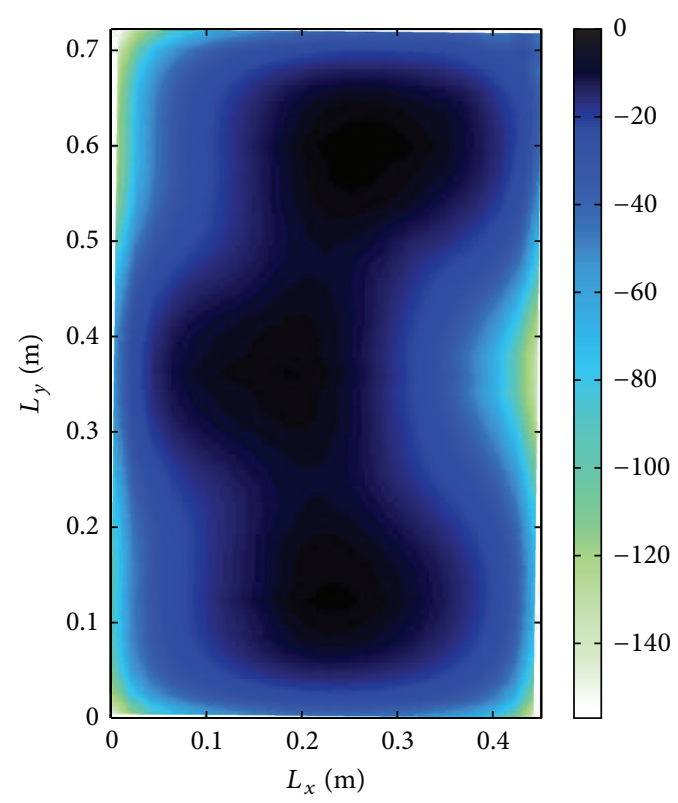

(b) Mode 3



(d) Mode 5

FIGURE 18: Plots of the WSSG field measured experimentally at the natural frequencies of modes 2-5.

but it was still able to cause minor distortions in the response at frequencies close to its natural frequency. Similar slight distortion may have happened with the fifth experimental mode, at $291 \mathrm{~Hz}$ in Figure 19, which was a clearly recognizable $(2,3)$ mode, predicted as the seventh mode in simulations (see Figure 20), but could have been slightly distorted by a nearby $(1,4)$ mode, predicted as the sixth. A clearly recognizable $(1$, 4) mode was not seen in the scans but may have still been present at small amplitude.

The mode shape at $365 \mathrm{~Hz}$ in Figure 19 was unrecognizable, although it may have been a highly distorted $(2,4)$ mode, predicted as the eighth mode in simulations. The analytical model predicted that a $(2,4)$ mode should appear after the $(2$, $3)$ mode, but a $(2,4)$ mode shape was indistinguishable in the SLDV scans.

Despite these differences, the experiments demonstrated several important results with regard to the use of WSSG experimentally. The first is that in Figures 6 and 19, when control was implemented using WSSG, both lines have the same general trends. They have significant attenuation at most peaks and minimal amplification at most off resonance frequencies. The experimental attenuation levels, however, 




FIGURE 19: Experimental control of the clamped plate over the frequency range from 60 to $370 \mathrm{~Hz}$.

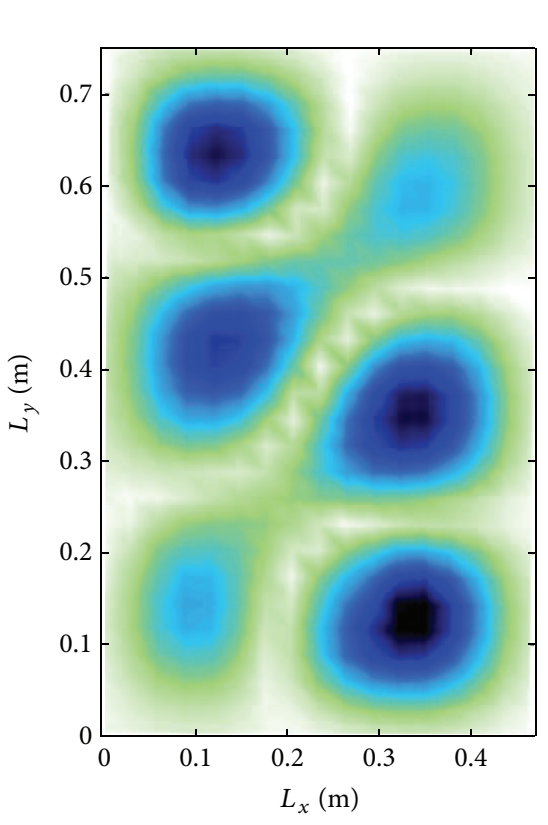

(a) Control off
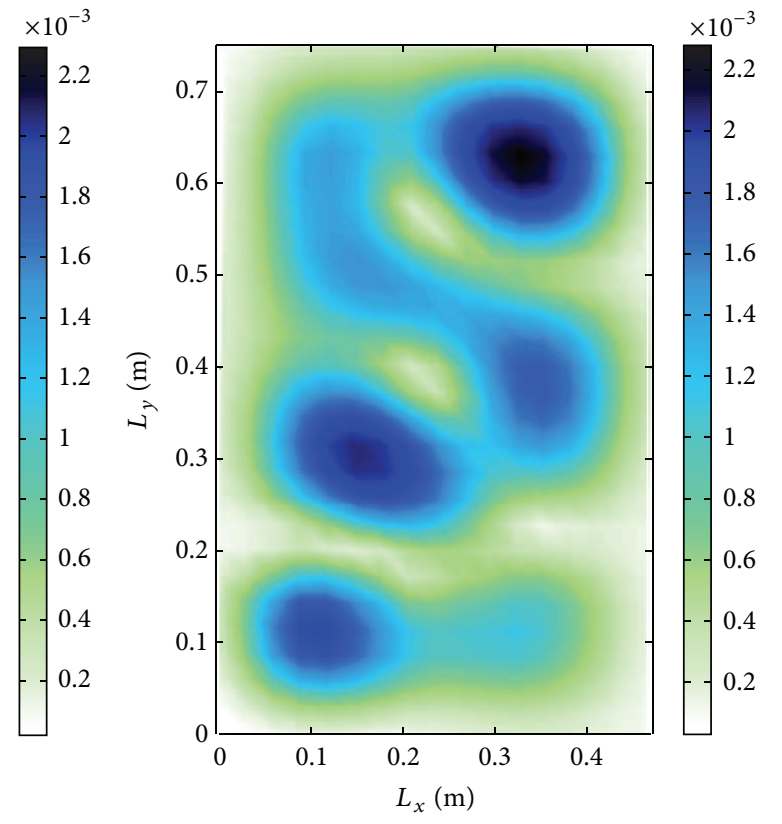

(b) Control on

FIgURE 20: The WSSG field with the plate excited at the fifth mode natural frequency, with control off and on.

were lower than the simulated values, likely due to the inherent difficulty in perfectly matching experiment to simulation, which was manifested by such things as noise in the signal, and overlapping structural modes which were not predicted by the simulations.

One resonance frequency of particular interest was the (2, 3) mode, which appeared as the fifth mode in experiments, where the experimental results show a minimal attenuation at the peak itself and amplification immediately following the peak, a different effect from the $19.7 \mathrm{~dB}$ of attenuation predicted by the simulations. In analyzing this mode it was found that it was highly distorted, causing several of the antinodes in the velocity field to have significantly lower amplitudes. This effect can be seen in Figure 20(a) which shows the squared velocities before control. When the mode was controlled, the four high antinodes were minimized, but the low ones were amplified, as seen in Figure 20(b), which shows the squared velocities of the $(2,3)$ mode after control. A similar effect occurred in the WSSG fields for this mode.

WSSG theory assumes that the WSSG field is uniform across the entire plate; thus minimizing one point will theoretically minimize WSSG across the entire plate. But if the WSSG field is not uniform to begin with, as in the case of the fifth experimental mode, then minimizing a single point may end up amplifying the WSSG field in areas where it originally was small. This was found to be the case in the fifth mode and the frequencies following it, where amplification occurred. It is suspected that these effects could be minimized if 
the mass and stiffness effects of the shakers could be negated. This could possibly be done by using smaller shakers, which would reduce the distortion from mass loading of the plate, allowing for a more uniform WSSG field.

\section{Conclusions}

A weighted sum of spatial gradients (WSSG) minimization quantity has been developed for a clamped flat rectangular plate following the method used previously to develop WSSG on a simply supported rectangular plate. An approximate solution for a rectangular flat plate clamped on all four sides, assuming that the shape functions for the flat plate were the product of clamped-clamped beam shape functions, has been used. This solution was used to derive analytical expressions for the weights in WSSG, which caused the field to become relatively uniform. The development of WSSG with the choice of weights was intended to create a measurement quantity which was insensitive to sensor location, to target and control the first four radiation mode shapes, and to be a metric that could be measured in a much simpler manner experimentally than previous minimization quantities.

Simulated results verified the hypothesized effects. While only measuring the acceleration at four closely spaced locations on the plate, improved control over volume velocity (which required 16-25 accelerometers, or geometry dependent sensors) was achieved. In particular the minimization quantity achieved improved control when compared to volume velocity at natural frequencies and modes higher than the fourth mode. This was due to the fact that WSSG could control the second, third, and fourth radiation modes, whereas volume velocity could not. The second, third, and fourth radiation modes have a greater contribution from structural modes with higher natural frequencies when compared to the first radiation mode, accounting for this improved control.

Also, the WSSG measurement location has been found to be relatively unimportant in simulations. This has been shown by placing the sensor in random locations and comparing the control using those locations. Control was most consistent above the fourth mode natural frequency, where more of the targeted radiation mode shapes become relevant.

Finally, to demonstrate the effectiveness of this WSSG control approach, experimental tests have been performed. The experimental results were similar to the simulation results, verifying that the simulation model was accurate and also confirming that the desired effects from the WSSG quantity do in fact occur.

From the observed effects it can be concluded that WSSG, which provides better control than volume velocity, also has several advantages in implementation. It requires fewer sensors, its physical measurement location is relatively geometry independent, and it works on multiple boundary conditions.

\section{Conflict of Interests}

The authors declare that there is no conflict of interests regarding the publication of this paper.

\section{References}

[1] J. D. Jones and C. R. Fuller, "Active control of sound fields in elastic cylinders by multicontrol forces," AIAA journal, vol. 27, no. 7, pp. 845-852, 1989.

[2] C. R. Fuller, "Active control of sound transmission/radiation from elastic plates by vibration inputs: I. Analysis," Journal of Sound and Vibration, vol. 136, no. 1, pp. 1-15, 1990.

[3] C. R. Fuller, C. H. Hansen, and S. D. Snyder, "Active control of sound radiation from a vibrating rectangular panel by sound sources and vibration inputs: an experimental comparison," Journal of Sound and Vibration, vol. 145, no. 2, pp. 195-215, 1991.

[4] F. Fahy and P. Gardonio, Sound and Structural Vibration, Academic Press, London, UK, 2nd edition, 2007.

[5] S. J. Elliott and M. E. Johnson, "Radiation modes and the active control of sound power," The Journal of the Acoustical Society of America, vol. 94, no. 4, pp. 2194-2204, 1993.

[6] T. C. Sors and S. J. Elliott, "Volume velocity estimation with accelerometer arrays for active structural acoustic control," Journal of Sound and Vibration, vol. 258, no. 5, pp. 867-883, 2002.

[7] P. Gardonio, Y.-S. Lee, S. J. Elliott, and S. Debost, "Analysis and measurement of a matched volume velocity sensor and uniform force actuator for active structural acoustic control," Journal of the Acoustical Society of America, vol. 110, no. 6, pp. 3025-3031, 2001.

[8] S. D. Snyder, N. Tanaka, and Y. Kikushima, "Use of optimally shaped piezo-electric film sensors in the active control of free field structural radiation, part 1: feedforward control," Transactions of the ASME, Journal of Vibration and Acoustics, vol. 117, no. 3, pp. 311-322, 1995.

[9] D. A. Manwill, J. M. Fisher, S. D. Sommerfeldt, K. L. Gee, and J. D. Blotter, "On the use of energy based metrics in active structural acoustic control," in Proceedings of the Meetings on Acoustics, vol. 9, Sydney, Australia, October 2010.

[10] J. M. Fisher, J. D. Blotter, S. D. Sommerfeldt, and K. L. Gee, "Development of a pseudo uniform structural quantity for use in active structural acoustic control of simply supported plates: an analytical comparison," The Journal of the Acoustical Society of America, vol. 131, pp. 3833-3840, 2012.

[11] D. R. Hendricks, W. R. Johnson, S. D. Sommerfeldt, and J. D. Blotter, "Experimental active structural acoustic control of simply supported plates using a weighted sum of spatial gradients," The Journal of the Acoustical Society of America, vol. 136, no. 5, pp. 2598-2608, 2014.

[12] W. R. Johnson, D. R. Hendricks, M. J. Anderson, J. D. Blotter, and S. D. Sommerfeldt, "Active structural acoustic control using a sum of weighted spatial gradients control metric," The Journal of the Acoustical Society of America, vol. 134, no. 5, pp. 41904190, 2013.

[13] S. S. Rao, Vibration of Continuous Systems, John Wiley \& Sons, Hoboken, NJ, USA, 2007.

[14] A. Leissa, Vibration of Plates, American Institute of Physics, 1993.

[15] C. C. Sung and C. T. Jan, "Active control of structurally radiated sound from plates," Journal of the Acoustical Society of America, vol. 102, no. 1, pp. 370-381, 1997.

[16] S. D. Sommerfeldt and P. J. Nashi, "An adaptive filtered-x algorithm for energy-based active control," The Journal of the Acoustical Society of America, vol. 96, no. 1, pp. 300-306, 1994. 

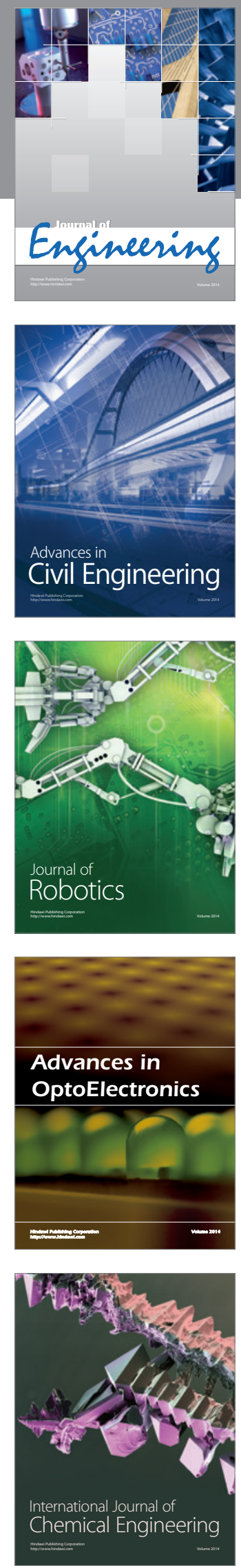



The Scientific World Journal
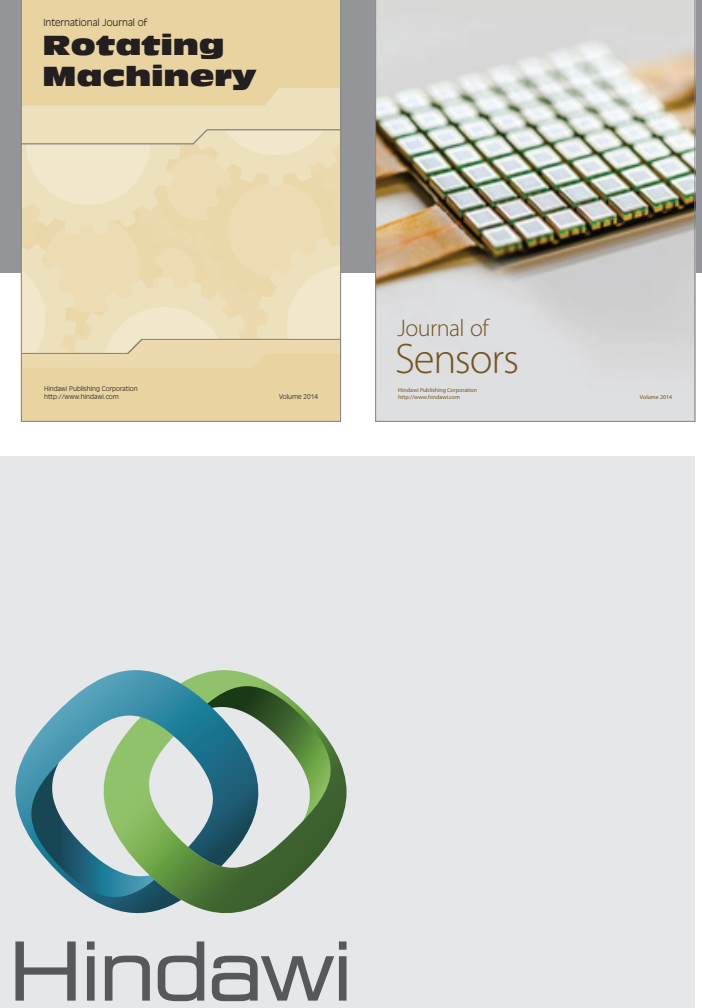

Submit your manuscripts at http://www.hindawi.com
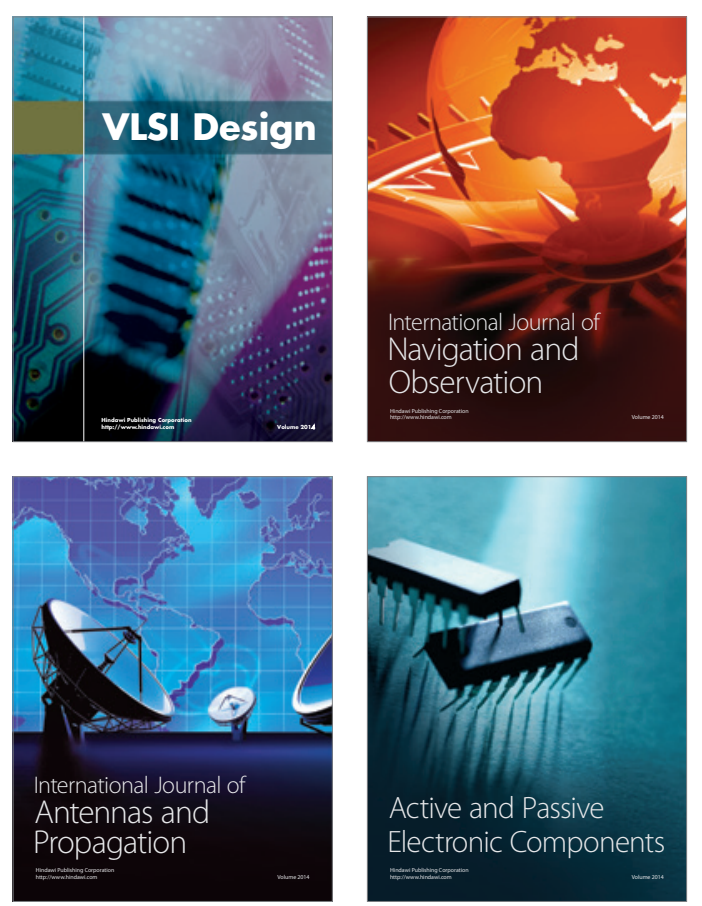
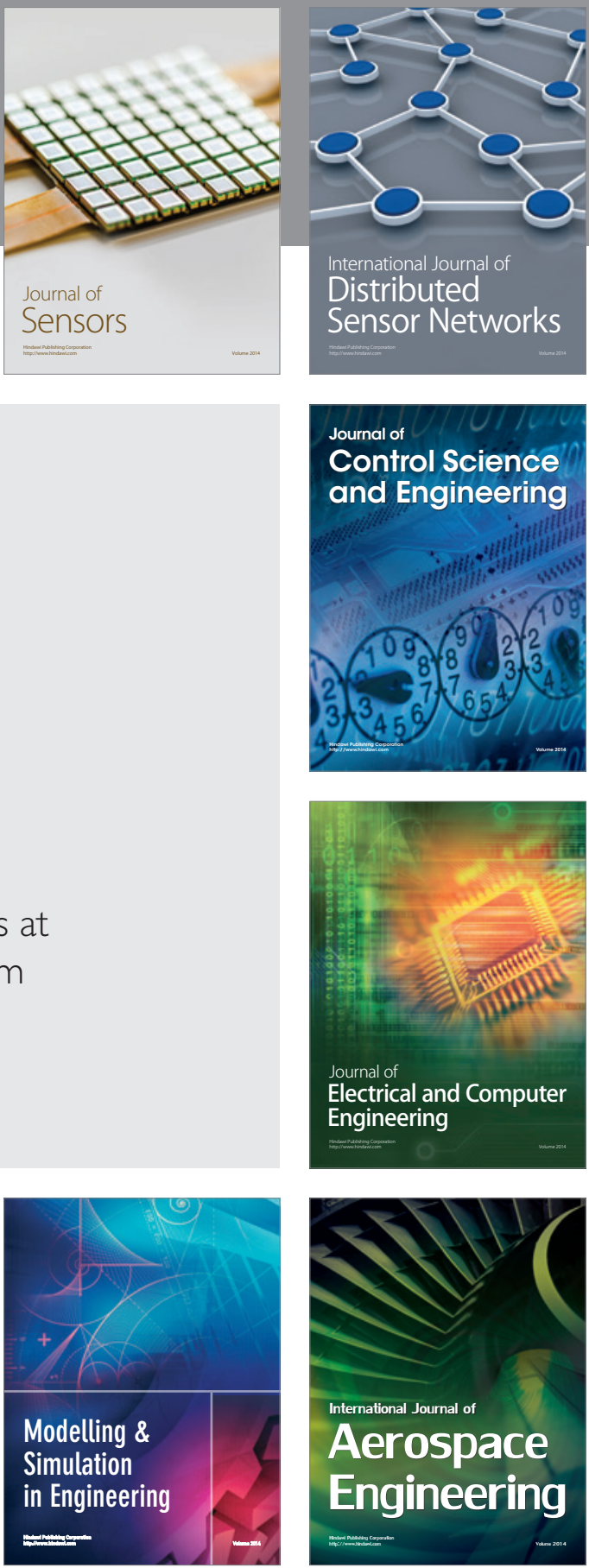

Journal of

Control Science

and Engineering
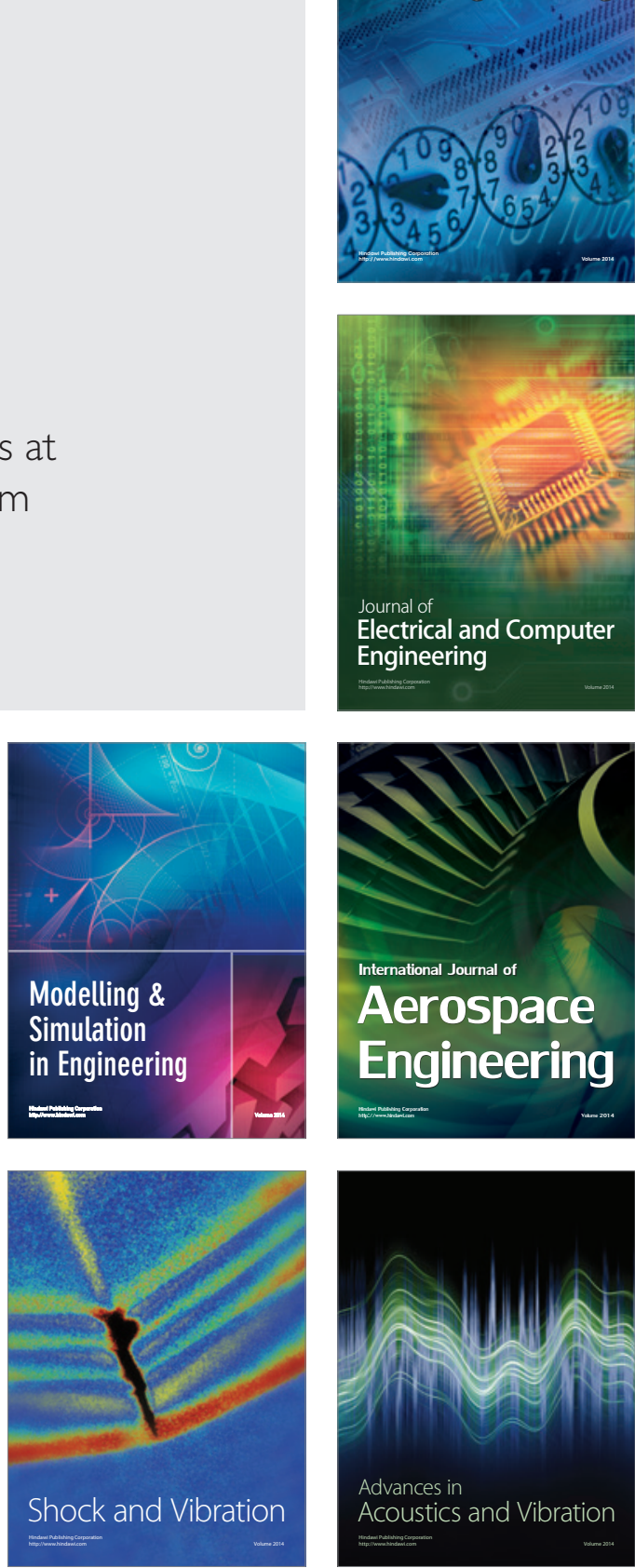